\title{
Retigeric acid B-induced mitophagy by oxidative stress attenuates cell death against prostate cancer cells in vitro
}

\author{
Yong-qing LIU ${ }^{1,2}$, Yuan $\mathrm{JI}^{2, \#}$, Xian-zhe $\mathrm{LI}^{1}$, Ke-li TIAN ${ }^{1}$, Charles YF YOUNG ${ }^{3}$, Hong-xiang LOU², Hui-qing YUAN ${ }^{1, *}$ \\ ${ }^{1}$ Department of Biochemistry and Molecular Biology, School of Medicine, Shandong University, Ji-nan 250012, China; ${ }^{2}$ Department of \\ Natural Product Chemistry, School of Pharmaceutical Sciences, Shandong University, Ji-nan 250012, China; ${ }^{3}$ Department of Urology, \\ Mayo Clinic College of Medicine, Mayo Clinic, Rochester, MN 55905, USA
}

\begin{abstract}
Aim: Retigeric acid B (RAB), a pentacyclic triterpenic acid from Lobaria kurokawae Yoshim, has been found to induce apoptosis in prostate cancer cells. The aim of this study was to investigate the roles of mitochondrial damage-caused mitophagy in RAB-induced prostate cancer cell death in vitro.

Methods: Human prostate cancer PC3 and LNCaP cells were tested. Cell viability was analyzed with MTT assay. Cell apoptosis, ROS level and mitochondrial transmembrane potential $(\mathrm{mt} \Delta \psi)$ were measured with flow cytometry. Autophagy- and apoptosis-related proteins were studied using Western blotting. GFP-LC3B puncta, mitochondrial swelling and mitophagy were examined morphologically. Quantitative RT-PCR was used to measure LC3B mRNA level, and siRNA was used to knock down LC3BII.

Results: In both PC3 and LNCaP cells, RAB (15 $\mu \mathrm{mol} / \mathrm{L})$ increased ROS accumulation and decreased $\mathrm{mt} \Delta \psi$ in a time-dependent manner. Furthermore, RAB induced mitochondrial swelling and mitophagy, significantly increased LC3B expression and conversion of LC3BI to LC3BII, and the elimination of mitochondria by LC3BII-containing autophagolysosomes. In addition, RAB suppressed the $\mathrm{PI} 3 \mathrm{~K} / \mathrm{Akt} / \mathrm{mTOR}$ pathway activation. Pretreatment of PC3 cells with autophagy inhibitor 3-MA ( $5 \mathrm{mmol} / \mathrm{L})$ or the lysosomal protease inhibitor CQ $(10 \mu \mathrm{mol} / \mathrm{L})$ significantly increased RAB-induced apoptosis. Similar results were obtained in RAB-treated PC3 cells with LC3B knocked down.
\end{abstract}

Conclusion: RAB induces mitochondrial damage and mitophagy that attenuates RAB-induced prostate cancer cell death. Thus, suppression of mitophagy might be a potential strategy for improving the chemotherapeutic effects of RAB.

Keywords: retigeric acid B; prostate cancer; reactive oxygen species (ROS); mitochondria; mitophagy; apoptosis

Acta Pharmacologica Sinica (2013) 34: 1183-1191; doi: 10.1038/aps.2013.68; published online 29 Jul 2013

\section{Introduction}

Autophagy is a catabolic pathway that maintains cellular homeostasis via the self-digestion of unwanted proteins and damaged or aging organelles, such as damaged mitochondria, by double-membrane autophagosomes ${ }^{[1,2]}$. In mammals, the Atg8 homolog LC3BI is conjugated with phosphatidylethanolamine, forming LC3BII and resulting in the maturation of autophagosomes, which associate with lysosomes to form autophagolysosomes ${ }^{[3]}$. When cells suffer extra- or intracellular stress, the level of autophagy could be remarkably activated as a cytoprotective response, resulting in adaptation and survival ${ }^{[4,5]}$, while autophagic cell death could occur with

\footnotetext{
\# Now in Pharmaceutical Sciences 2010, School of Pharmaceutical Sciences, Shandong University, Ji-nan 250012, China

* To whom correspondence should be addressed.

E-mail lyuanhq@sdu.edu.cn

Received 2013-02-14 Accepted 2013-04-28
}

dysregulated or excessive autophagy.

DJ-1 is a member of the peptidase C56 family of proteins and was originally identified as a novel oncogene that works in collaboration with ras ${ }^{[6]}$. It is generally known that loss-offunction DJ-1 mutations can cause early-onset Parkinson's disease. DJ-1 acts as a redox-sensitive chaperone and a sensor for oxidative stress ${ }^{[7]}$; it also protects cells against oxidative stress during neuronal injury ${ }^{[8,9]}$. Furthermore, DJ-1 protects several types of cancer cells, including leukemic ${ }^{[10]}$, lung carcinoma ${ }^{[11]}$, and breast cancer ${ }^{[12]}$ cells, from oxidative stress-induced apoptosis. The oxidative stress-induced early translocalization of DJ-1 into mitochondria is closely associated with its cytoprotective activity; DJ-1 is mainly localized in the cytoplasm, but under oxidative stress, it is translocated into mitochondria ${ }^{[13]}$. It has been reported that DJ-1 maintains mitochondrial functions during oxidative stress ${ }^{[14,15]}$, and the loss of DJ-1 leads to alterations in mitochondrial phenotypes such as a reduction in 
membrane potential and an increase in fragmentation; damaged mitochondria then promote mitochondrial clearance via the mitophagy pathway ${ }^{[14]}$. These events are thought to be a protective mechanism against oxidative stress.

Recent studies have demonstrated that autophagy, including mitophagy, is often stimulated in tumor cells in response to multiple forms of cellular stress induced by chemotherapeutic agents ${ }^{[16-18]}$. In fact, the mode of action of some anticancer drugs from natural materials such as paclitaxel and camptothecin ${ }^{[19,20]}$ involves the induction of autophagy, which is important for the regulation of cancer development and progression and for determining the response of tumor cells to anticancer therapy. Driven by these findings, we expect to find novel natural agents that target autophagy. Retigeric Acid $B(R A B)$ is a natural pentacyclic triterpenic acid isolated from Lobaria kurokawae Yoshim. Although a role for RAB in anti-tumor activity has been reported ${ }^{[21,22]}$, the mechanisms for $\mathrm{RAB}$ in the regulation of the function of mitochondria and mitophagy in cancer cells have not been described. Here, we found that RAB induced mitochondrial depolarization and a reduction in DJ-1 expression followed by protective autophagy via the elimination of mitochondria by LC3BII-containing autophagolysosomes (mitophagy), which attenuates apoptosis in prostate cancer $(\mathrm{PCa})$ cells.

\section{Materials and methods}

\section{Cell culture and treatments}

Human prostate cancer PC3 and LNCaP cells [American Type Culture Collection (ATCC)] were cultured in RPMI-1640 medium (HyClone, Thermo Fisher Scientific, USA) supplemented with $10 \%$ fetal bovine serum (HyClone) and antibiotics. The human glioma cancer cell line U87, which stably expresses the GFP-LC3 protein, was a kind gift from Bing YAN (Shandong University) and was maintained in DMEM medium (HyClone) supplemented with $10 \%$ fetal bovine serum (HyClone) and antibiotics. The cells were maintained in a humidified incubator with $5 \% \mathrm{CO}_{2}$ at $37^{\circ} \mathrm{C}$.

$\mathrm{RAB}$ was isolated from Lichen L kurokawae as previously described ${ }^{[21,22]}$. RAB was prepared in dimethyl sulfoxide (DMSO) at $10 \mathrm{mmol} / \mathrm{L}$ as a stock solution, stored at $-20^{\circ} \mathrm{C}$ and diluted according to experimental requirements.

\section{Cell viability and cell death assay}

MTT was used to quantitate cell proliferation and cytotoxicity. After required pre-treatment, PC3 or LNCaP cells were exposed to RAB or vehicle for $24 \mathrm{~h}$. Cell proliferation was detected with the 3-[4,5-dimethylthiazol-2-yl]-2,5-diphenyltetrazolium bromide (MTT; Sigma-Aldrich, USA) colorimetric assay $^{[21]}$.

For the cell death assay, RAB-treated PC3 cells were collected, and apoptosis was determined with $10 \mu \mathrm{g} / \mathrm{mL}$ propidium iodide staining (PI; Sigma-Aldrich, USA) and flow cytometry using a Becton Dickinson FACScan.

In some experiments, cells were exposed to $5 \mathrm{mmol} / \mathrm{L}$ of the autophagy inhibitor 3-methyladenine (3-MA; Sigma-Aldrich, USA) for $2 \mathrm{~h}, 10 \mu \mathrm{mol} / \mathrm{L}$ of the lysosomal protease inhibitor chloroquine (CQ; Sigma-Aldrich, USA) for $2 \mathrm{~h}$ or $2 \mathrm{mmol} / \mathrm{L}$ of the antioxidant $\mathrm{N}$-acetylcysteine (NAC; Sigma-Aldrich, USA) for $1 \mathrm{~h}$ before treatment with RAB. The cell proliferative response to the chemicals was then analyzed by MTT assay or PI exclusion determination.

\section{Transmission electron microscopy analysis}

PC3 cells were treated with $15 \mu \mathrm{mol} / \mathrm{L} \mathrm{RAB}$ for the indicated time. Cells were fixed with $2.5 \%$ glutaraldehyde in 0.1 $\mathrm{mmol} / \mathrm{L}$ phosphate buffer $(\mathrm{pH} 7.4)$ and rinsed with phosphate buffer, followed by incubation in 1\% osmium tetroxide. After a series of dehydration, the cell pellets were embedded in epon resin, and ultrathin sections were cut and stained with $2 \%$ uranyl acetate. A JEM-1400 transmission electron microscope (JEOL, Japan) was used to analyze changes in the cellular ultrastructure.

\section{Immunofluorescence staining for LC3B}

For immunofluorescence analysis of RAB-induced LC3B in PC3 cells, a confocal laser-scanning microscope (Carl Zeiss, LSM780, Germany) was used; the procedures have been previously described $^{[21]}$. An antibody directed against LC3B was used for the immunofluorescence experiments, and nuclei were stained with DAPI (Sigma-Aldrich, USA).

\section{Microscopy for GFP-LC3B puncta}

To determine autophagy in U87 cells, which stably express the GFP-LC3B protein, we exposed U87 cells to $15 \mu \mathrm{mol} / \mathrm{L}$ RAB for $12 \mathrm{~h}$, and the nuclei were then stained with Hoechst 33342 (Sigma-Aldrich, USA) for $15 \mathrm{~min}$. The GFP-LC3B puncta in response to $R A B$ or vehicle treatment were observed with a fluorescence microscope (Nikon, Japan). Vehicle-treated cells served as a control.

\section{Quantitative RT-PCR analysis}

Total RNA from PC3 and LNCaP cells treated with RAB for the indicated time periods were extracted with the RNAiso plus kit (Takara, Japan). For quantitative RT-PCR (qRT-PCR), cDNA was synthesized using the ReverTra Ace qPCR RT Kit (Toyobo, Japan). qRT-PCR was performed with a Realtime PCR System (Eppendorf International, Germany). The sequences of the primers for $\mathrm{LC} 3 \mathrm{~B}$ and GAPDH were as follows: LC3B, 5' -GAAGATGTCCGACTTATTCGAGAG-3' (forward) and 5'-ACTCTCATACACCTCTGAGATTGG-3' (reverse) and GAPDH, 5'-TGGTCACCAGGGCTGCTT-3' (forward) and 5'-AGCTTCCCGTTCTCAGCCTT-3' (reverse), respectively. The mRNA level of $\mathrm{LC} 3 \mathrm{~B}$ was normalized to the level of GAPDH.

\section{RNA interference of LC3B}

PC3 cells were transiently transfected with siRNA duplex oligonucleotides targeting LC3B (GenePharmcon, China) using Lipofectamine ${ }^{\mathrm{TM}} 2000$ (Invitrogen, USA). The LC3B siRNA sequences were as follows: sense: $5^{\prime}$ - GCACCUUCGAACAAAGAGUTT-3' and antisense: 5'-ACUCUUUGUUCGAAGGUGCTT-3'. The scramble siRNA sequences were as 
follows: sense: 5'-UUCUCCGAACGUGUCACGUTT-3' and antisense: 5'-ACGUGACACGUUCGGAGAATT-3'. After 24 or $48 \mathrm{~h}$ of transfection, the cells were treated with RAB or vehicle for an additional $24 \mathrm{~h}^{[21]}$, and the effects of RAB on the conditioned cells were evaluated using Western blotting and cell survival assay.

\section{Western blotting assay}

After treatment as indicated, cell lysates were prepared with RIPA buffer, and the procedures for Western blotting have been described previously ${ }^{[23]}$. Blots were incubated with the following primary antibodies: LC3B, which was purchased from Novus Biologicals, Inc, USA; Atg5, mTOR, phosphomTOR (Ser2448), Akt, phospho-Akt (Ser473), phospho-p70S6K (Thr389), p62/SQSTM1, DJ-1, and Beclin1, which were from Cell Signal Technology, Inc, USA; Glyceraldehyde-3-phosphate dehydrogenase (GAPDH) and poly(ADP-ribose) polymerase (PARP), which were from Santa Cruz Biotechnology, Inc, USA. GAPDH served as a protein loading control.

Mitochondrial transmembrane potential $(\mathrm{mt} \Delta \Psi)$ and reactive oxygen species (ROS) detection

PC3 or LNCaP cells incubated with $15 \mu \mathrm{mol} / \mathrm{L}$ RAB for different times were collected, and the $\mathrm{mt} \Delta \psi$ was measured (mitochondrial membrane potential assay kit with JC-1, Beyotime, China) by flow cytometry. The production of ROS induced by RAB was detected by flow cytometry using the fluorescent dye hydroethidine 2', $7^{\prime}$-dichlorodihydrofluorescein diacetate (H(2)DCFDA, Molecular Probes, Sigma-Aldrich, USA).

\section{Statistical analysis}

The data were presented as the mean \pm SD of at least three independent experiments. Student's $t$-test was used to determine significant differences between treated and control groups, and a $P<0.05$ was considered statistically significant.

\section{Results}

\section{RAB decreases $m t \Delta \psi$ in PCa cells}

Because RAB has been found to exert cytotoxic effects on human PCa cells with the induction of a higher ratio of Bax/ $\mathrm{Bcl}-2$ proteins and the activation of caspase- $3^{[22]}$, we initiated our study to determine whether $\mathrm{RAB}$ was also involved in mitochondrial damage. The $\mathrm{mt} \Delta \psi$, an indicator of the energetic state of mitochondria, reflects the activity of mitochondrial proton pumps, electronic transport systems, and the activation of the mitochondrial permeability transition (MPT) ${ }^{[24]}$. Therefore, to evaluate whether RAB interfered with mitochondrial function, we first examined its effects on $\mathrm{mt} \Delta \Psi$ in PCa cells with the probe JC-1. In cells with mitochondria having a high $\mathrm{mt} \Delta \psi$, JC-1 formed orange-red fluorescent J-aggregates (R1 regions of Figure 1A), while in cells with depolarized or damaged mitochondria, the sensor dye appeared as green fluorescent monomers (R2 regions of Figure 1A). As shown in Figure $1 \mathrm{~A}, \mathrm{mt} \Delta \psi$ decreased in response to $\mathrm{RAB}$ in a time-
A RAB
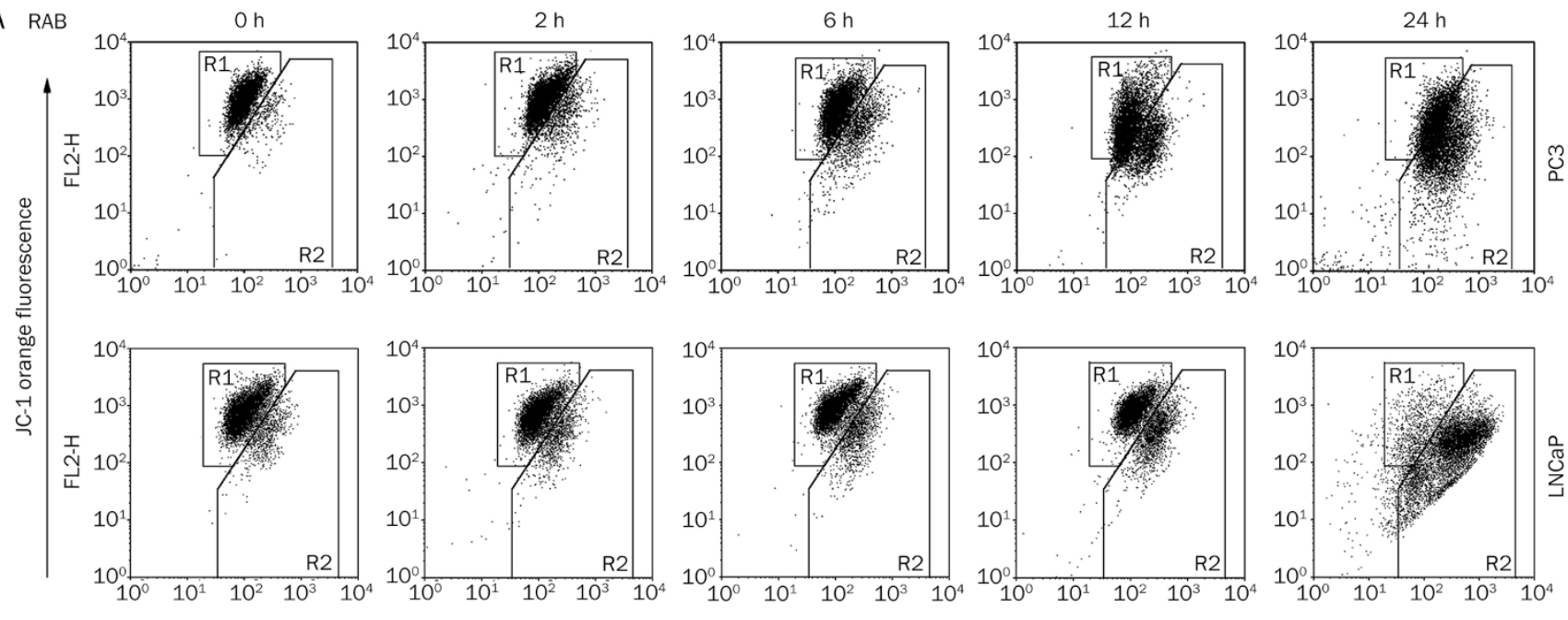

FL1-H
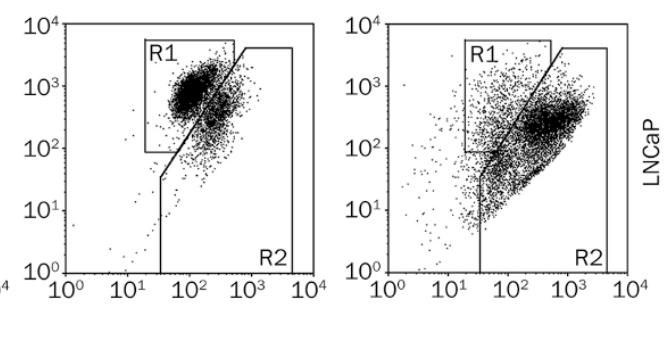

JC-1 green fluorescence

B

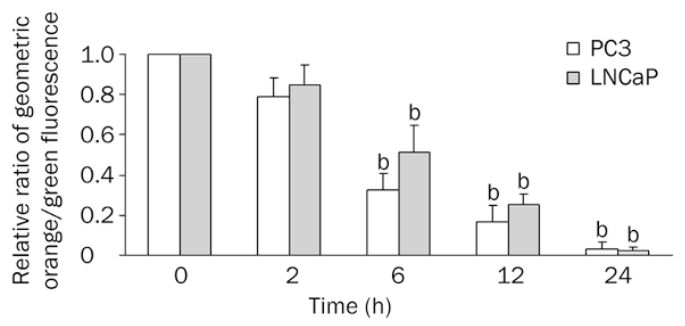

Figure 1. Effect of RAB on $m t \triangle \psi$. (A) PC3 and LNCaP cells were treated with $15 \mu \mathrm{mol} / \mathrm{L}$ RAB for the indicated time and collected, and the $\mathrm{mt} \Delta \psi$ was evaluated by labeling cells with the JC-1 dye and analyzing by flow cytometry. A representative dot plot (J-aggregated orange fluorescence and monomer green fluorescence) is shown. Cell populations with higher and lower J-aggregated staining were gated as R1 and R2, respectively. (B) Statistical analysis of the relative ratio of geometric orange/green fluorescence. ${ }^{\mathrm{b}} \mathrm{P}<0.05$ vs the RAB-untreated control group. 
dependent manner. The relative ratio of $\mathrm{R} 1 / \mathrm{R} 2$ dots is represented in Figure 1B. Cells treated with $15 \mu \mathrm{mol} / \mathrm{L}$ RAB for $6 \mathrm{~h}$ showed a significant change, including an $\sim 20 \%$ decrease in PC3 cells and an $\sim 15 \%$ decrease in LNCaP cells $(P<0.05)$, and those quantities continued to decline for $24 \mathrm{~h}$ following $\mathrm{RAB}$ treatment, with a reduction of over $90 \%$ in PC3 and LNCaP cells $(P<0.05)$. Thus, the RAB-induced $m t \Delta \psi$ decrease indicates that RAB damaged mitochondria in PCa cells.

RAB induces the generation of ROS and a decrease of DJ-1 in PCa cells

To confirm that mitochondria were involved in mechanisms of cellular processes affected by RAB, the mitochondrial production of ROS was also assessed using the fluorescent probe $\mathrm{H}(2)$ DCFDA. The intracellular accumulation of ROS induced by $\mathrm{RAB}$ was determined by calculating the geometric mean $\left(G_{\text {mean }}\right)$ of fluorescence intensity in the affected cells with flow cytometry. In agreement with the changes in mitochon- drial depolarization, time kinetic studies (Figure 2A and 2B) revealed that $15 \mu \mathrm{mol} / \mathrm{L}$ RAB time-dependently increased ROS production in PC3 and LNCaP cells. As summarized in Figure 2C, the significant increase in ROS production began at $6 \mathrm{~h}$ in PC3 cells and at $12 \mathrm{~h}$ in LNCaP cells $(P<0.05)$, peaking at 24 h compared with the vehicle control.

To determine whether RAB-induced ROS production is involved in anti-tumor activity, we tested the scavenging effect of the antioxidant NAC on RAB-induced ROS generation and its anti-tumor function. As shown in Figure 2D, RAB significantly increased the generation of ROS in cells after a $12 \mathrm{~h}$ incubation. In addition, NAC prominently reduced the amount of endogenous and RAB-induced ROS, and at the same time, NAC partially blocked the RAB-mediated inhibitory effect on cell proliferation at $12 \mathrm{~h}(P<0.05)$.

DJ-1 acts as a redox-sensitive chaperone and sensor for oxidative stress $^{[7]}$, and it is known to protect cells against oxidative stress as an antioxidant because its cysteine residues

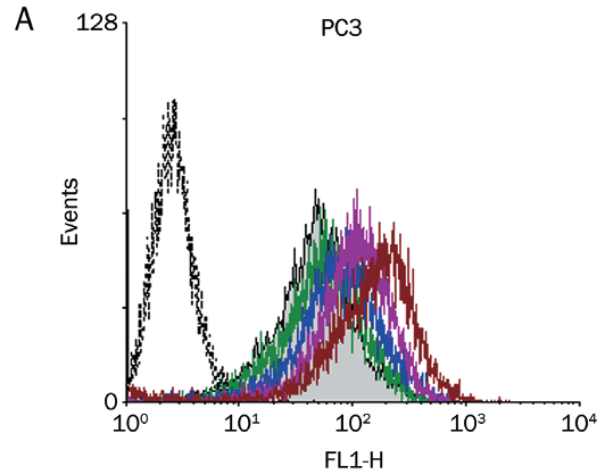

C
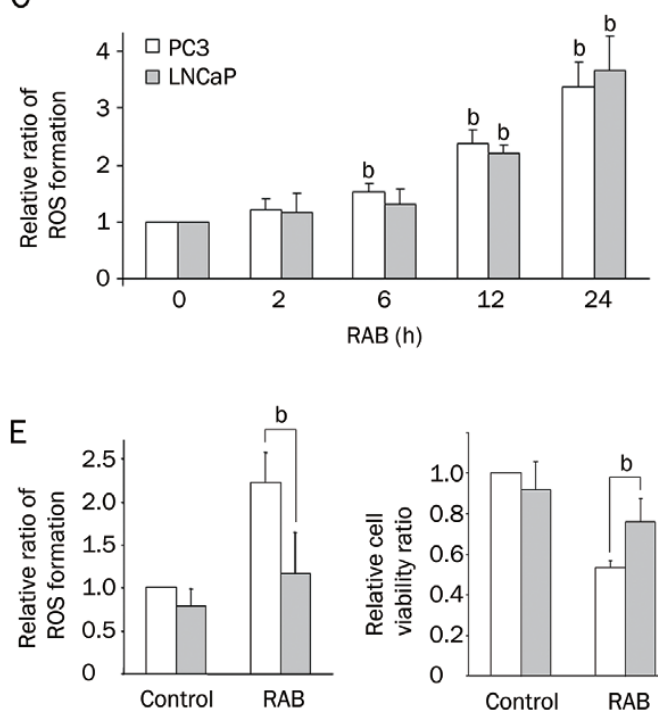

B

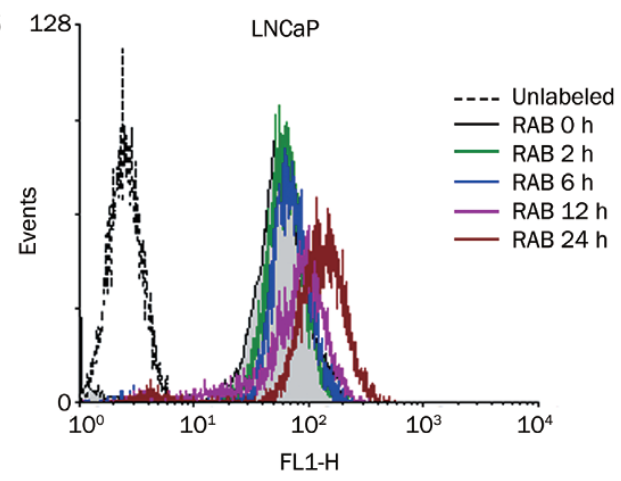

D

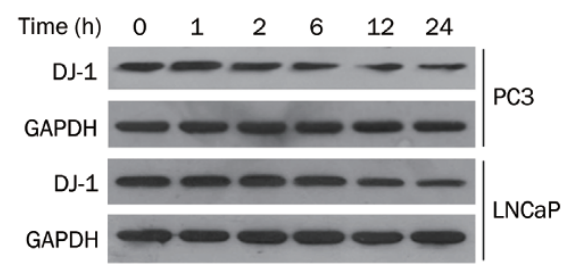

Figure 2. Effect of RAB on the generation of ROS and expression of DJ-1. (A and B) PC3 and LNCaP cells exposed to $15 \mu \mathrm{mol} / \mathrm{L}$ RAB for the indicated time were collected, stained with $\mathrm{D}(2)$ CFHDA and detected by flow cytometry. (C) Statistical analysis of the relative ratio of ROS formation. ${ }^{\mathrm{b}} P<0.05$ versus the RAB-untreated control group. (D) Effect of the antioxidant NAC on RAB-induced ROS generation. PC3 cells were pretreated with 2 mmol/L NAC or vehicle for $1 \mathrm{~h}$ and then incubated with RAB in the presence or absence of NAC for $12 \mathrm{~h}$. ROS production and cell viability were then assessed. ${ }^{\mathrm{b}} \mathrm{P}<0.05$ vs the NAC-untreated control group. (E) Western blotting analysis for expression of the DJ-1 protein. PC3 and LNCaP cells were treated with 15 $\mu \mathrm{mol} / \mathrm{L}$ RAB for different time as indicated. GAPDH served as a loading control. 
are sensitive to oxidative stress ${ }^{[14,15]}$. To investigate the effect of RAB on DJ-1 expression, we analyzed the time-dependent expression changes in the DJ-1 protein level with RAB treatment. Our results demonstrated that RAB slightly enhanced DJ-1 expression before $6 \mathrm{~h}$ and then remarkably decreased the expression level at $24 \mathrm{~h}$ (Figure 2E). Together, these data demonstrated that RAB induced ROS production in PCa cells, particularly at a late stage, which corresponds to down-regulated DJ-1. Thus, a massive loss of DJ-1 could not protect cells from RAB by inhibiting mitochondrial impairment.

\section{RAB stimulates mitophagy in PCa cells and inhibits the PI3K/ Akt/mTOR pathway}

Because mitochondrial depolarization and the loss of protection against oxidative stress were able to cause severe mitochondrial damage and fragmentation, which was followed by the anchoring of LC3BII autophagolysosomes to mitochondrial membranes (mitophagy) ${ }^{[1,14]}$, we examined whether RAB could induce mitophagy. First, we visualized the colocalization of mitochondria and autophagolysosomes by electron microscopy of RAB-treated PC3 cells and dramatic mitochondrial swelling at $12 \mathrm{~h}$ and $24 \mathrm{~h}$ (Figure 3A). We further confirmed RAB-induced autophagy by detecting the accumulation of the autophagy marker proteins LC3B nd lipidated LC3BII. As shown in Figure 3B, autophagic flux by RAB was observed at $2 \mathrm{~h}$ and persisted until $24 \mathrm{~h}$, maintaining high levels for $12-24 \mathrm{~h}$ as indicated by the increased processing of LC3B to LC3BII. The sequestome-1 protein (p62/SQSTM1), which was suggested to directly interact with LC3B and become degraded by autophagy, was considerably reduced after $2 \mathrm{~h}$ in response to RAB. The levels of Beclin 1 and Atg 5 were slightly increased at $24 \mathrm{~h}$. Compared with LC3BII and p62, the cleavage of PARP $(89 \mathrm{kDa})$ occurred much later and was significant after $12 \mathrm{~h}$. RAB-induced autophagy was also determined by the number of punctate dots comprising LC3B protein in PC3 cells (Figure 3C) and a green fluorescent protein-tagged form of LC3B (GFP-LC3B) in U87 cells with stable GFP-LC3B expression (Figure 3D). RAB-treated cells displayed significantly punctate green fluorescence in PC3 and U87 cells, while vehicle-treated cells were observed with diffuse green fluorescence. We also tested the mRNA level of LC3B, which was increased in a time-dependent manner by RAB-treatment in PC3 and LNCaP cells ( $P<0.05$ after $2 \mathrm{~h}$ ) (Figure 3E).

The PI3K/Akt/mTOR pathway is one of the major signaling pathways activated in cancer cells, and compelling evidence has indicated that inhibition of this pathway is associated with the initiation of autophagy in cancer cells ${ }^{[2]}$. Thus, we investigated whether $\mathrm{RAB}$ also triggers autophagy through the inhibition of PI3K/Akt/mTOR signaling. As shown in Figure $3 \mathrm{~F}$, treatment with RAB caused a significant decrease in the phosphorylation (at Ser473) of Akt after $6 \mathrm{~h}$, with a continuing decline until $24 \mathrm{~h}$, which corresponded with an obvious accumulation of LC3BII after $6 \mathrm{~h}$, as shown in Figure 3B. Moreover, we investigated the effect of RAB-treatment on mTOR activity. The exposure of PC 3 cells to RAB resulted in a time-dependent decrease in the phosphorylation of mTOR, yet the total mTOR remained unchanged. Similarly, phosphop70S6K, the downstream effector of mTOR, was dramatically inhibited by RAB. Therefore, the results revealed that the suppression of PI3K/Akt/mTOR signaling was involved in RABtriggered autophagy in PCa cells.

\section{Inhibition of autophagy potentiates RAB-induced cell death}

To assess whether the inhibition of autophagy could affect the cytotoxicity of $\mathrm{RAB}$, cell viability and cell death were analyzed by pretreating PC3 cells with the well-known autophagy inhibitor 3-MA ${ }^{[26]}$ or the lysosomal protease inhibitor $\mathrm{CQ}^{[27]}$. As shown in Figure 4A, 3-MA blocked the expression of LC3B, while CQ promoted LC3BII accumulation. Treatment of cells with RAB led to elevated processing of LC3BI to LC3BII, whereas this effect was significantly blocked in the presence of 3-MA and was accompanied by an increase in cleaved PARP $(89 \mathrm{kDa})$. In addition, combined treatment with $\mathrm{RAB}$ and 3-MA or CQ led to more a pronounced inhibition of cell viability (Figure 4B; $P<0.05$ for doses between $5-20 \mu \mathrm{mol} / \mathrm{L}$ ) and synergistically augmented the pro-apoptotic effects (Figure 4C), as detected by the MTT and PI assays, respectively.

We further investigated the role of LC3B, a crucial component involved in autophagic vesicle nucleation ${ }^{[1,4]}$, in the mode of action of RAB-induced autophagy. Knockdown of the endogenous expression of LC3B by LC3B-targeting siRNA resulted in a low level of LC3B (Figure 4D) and dramatically increased cleaved PARP (89 kDa) (Figure 4D). Accordingly, the knockdown of LC3B enhanced RAB-mediated cell death, as observed by a decrease in cell viability (Figure $4 \mathrm{E}$ ) and a higher percentage of apoptotic cells (Figure 4F). Thus, the results revealed that $\mathrm{RAB}$ promoted an autophagic process, which plays an early protective role that might delay PCa cell death.

\section{Discussion}

In this study, we first identified the potential mechanisms involved in the regulation of mitophagy by RAB treatment. Our results demonstrated that RAB time-dependently induced the generation of ROS and mitochondriotoxic effects, as demonstrated by mitochondrial membrane depolarization, swelling, the generation of ROS, and a decrease in the antioxidative protein DJ-1 in the prostate cancer cells PC 3 and LNCaP. To further elucidate the mode of action of $\mathrm{RAB}$, we assessed the role of mitophagy in RAB-induced mitochondrial damage. We found that RAB induced LC3B lipidation to form LC3B-II and mediated protective autophagy by inducing mitophagy. Moreover, blockade of the activation of PI3K/Akt/mTOR signaling also mediated RAB-triggered autophagy in PCa cells.

Reactive oxygen species (ROS) are the by-products of cellular metabolism and are primarily generated in the mitochondria. During oxidative stress, the level of ROS is elevated. In cases where the formation of ROS overwhelms its antioxidant capacity, the extra ROS is likely to damage mitochondria ${ }^{[28,29]}$ and is usually manifested by depolarization of the mitochondrial membrane such as that stimulated by RAB treatment. Accumulating data have recently revealed that massive ROS 
A

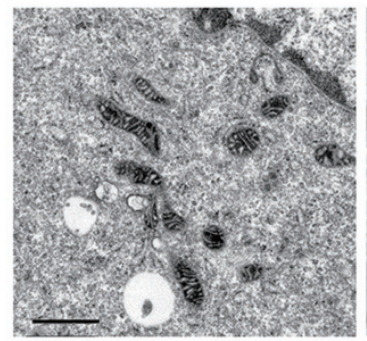

B

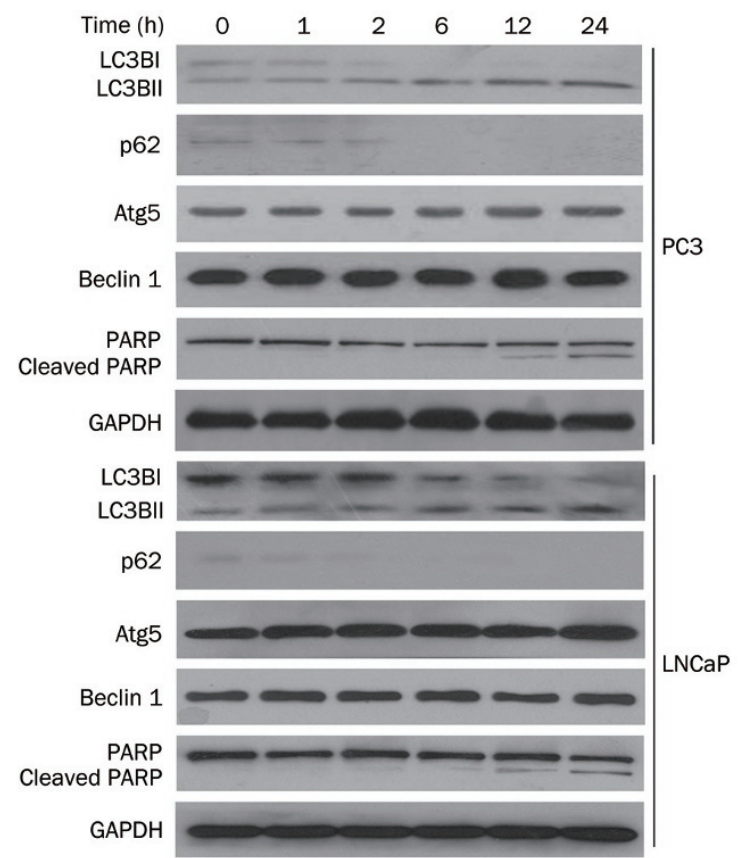

D
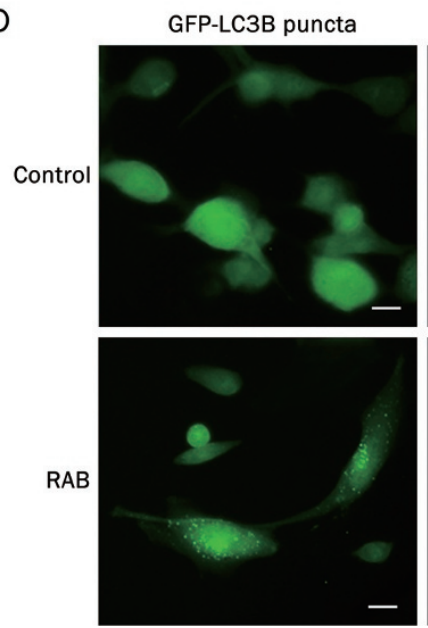

RAB $6 \mathrm{~h}$

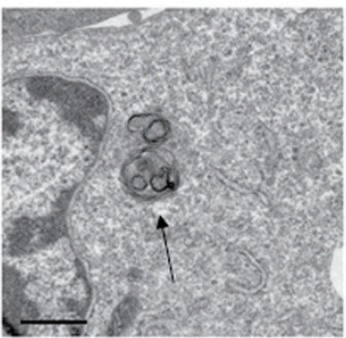

$2 \quad 6 \quad 12 \quad 24$

p62

GFP-LC3B puncta
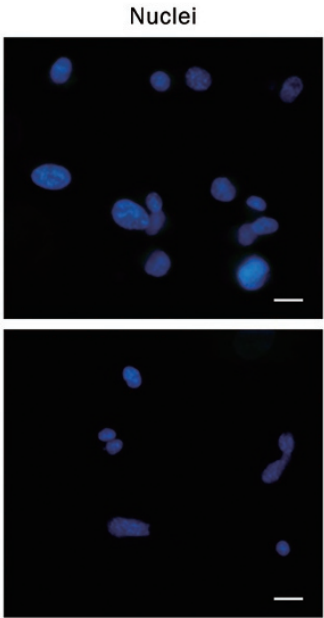

RAB $12 \mathrm{~h}$

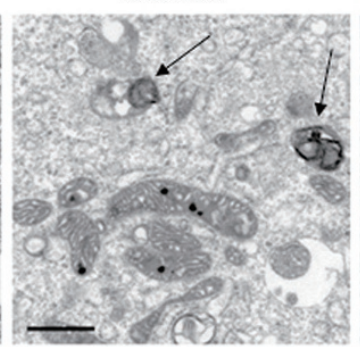

C
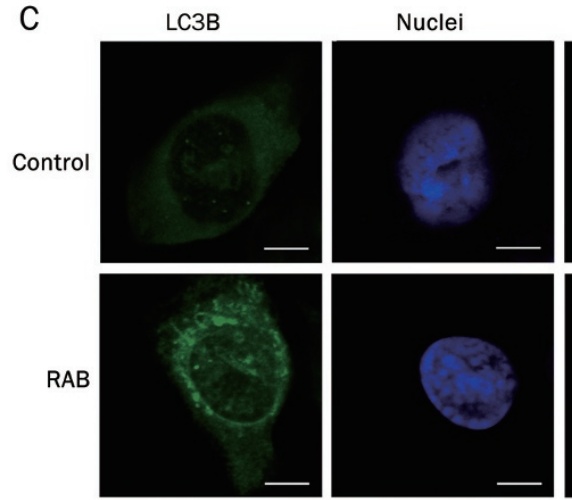

E

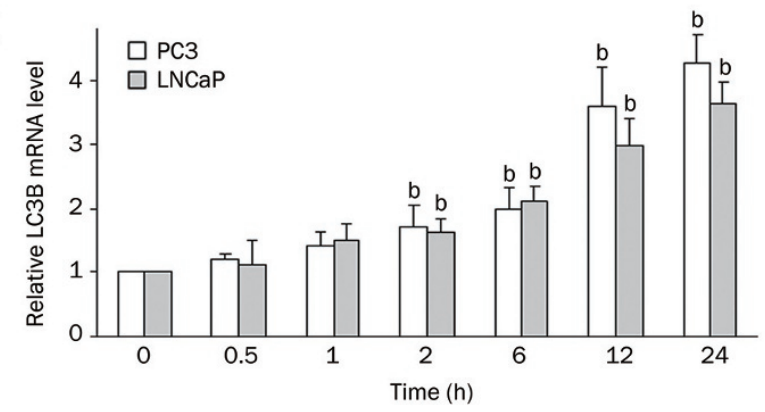

F

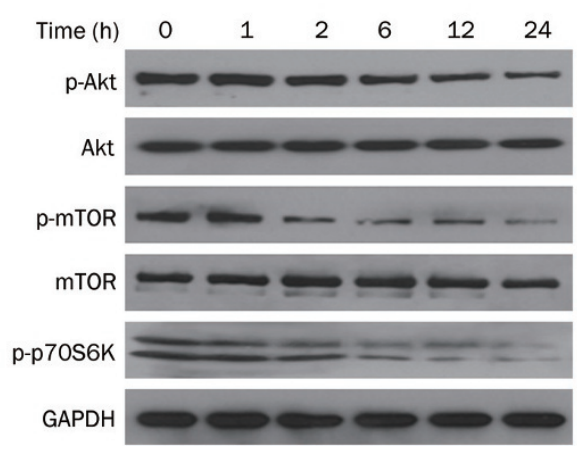

Figure 3. RAB promoted mitophagy and inactivated the PI3K/Akt/mTOR pathway in PCa cells. (A) Electron micrographs show swelling mitochondria and mitophagy induced by RAB. PC3 cells were treated with $15 \mu \mathrm{mol} / \mathrm{L}$ RAB for different time. The arrows in the electron micrograph represent autophagosomes. Scale bar, $1 \mu \mathrm{m}$. (B) RAB induced LC3B expression and conversion. Total lysates from cells treated with $15 \mu \mathrm{mol} / \mathrm{L}$ RAB for 12 and $24 \mathrm{~h}$ were used for Western blotting analysis to detect the levels of LC3B, p62, Atg5, Beclin 1, PARP, and GAPDH (loading control). (C) Immunofluorescence analysis of the LC3B punctate expression induced by RAB. Cells were treated with $15 \mu$ mol/L RAB for $12 \mathrm{~h}$, and LC3B was immunostained with nuclei staining performed with DAPI. Scale bar, $5 \mu \mathrm{m}$. (D) GFP-LC3B puncta analysis of RAB-induced autophagy. Stable GFPLC3B-expressing U87 cells were treated with RAB for $12 \mathrm{~h}$. LC3B puncta were observed by fluorescence microscopy with nuclei staining performed with Hoechst 33342. Scale bar, $10 \mu \mathrm{m}$. (E) RAB up-regulated LC3B mRNA level in PC3 and LNCaP cells, as detected by qRT-PCR assay. ${ }^{\mathrm{b} P}<0.05$ versus the RAB-untreated control group. (F) Western blotting analysis of the expression of proteins involved in the PI3K/Akt/mTOR pathway. PC3 cells were treated with $15 \mu \mathrm{mol} / \mathrm{L}$ RAB for the indicated time. GAPDH served as a loading control. 
A

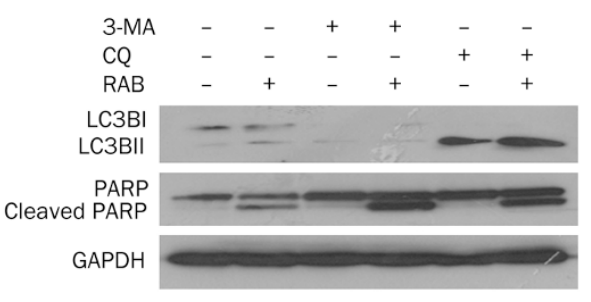

B

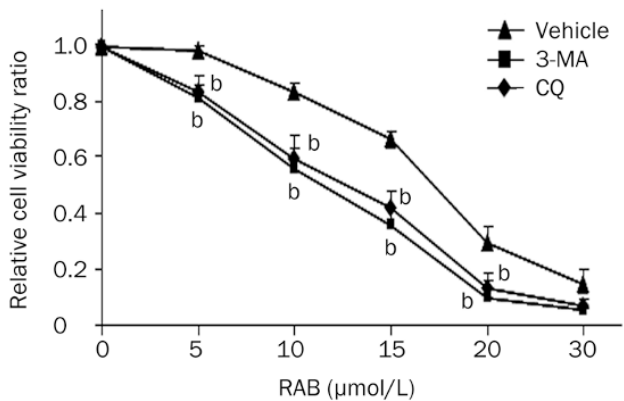

C
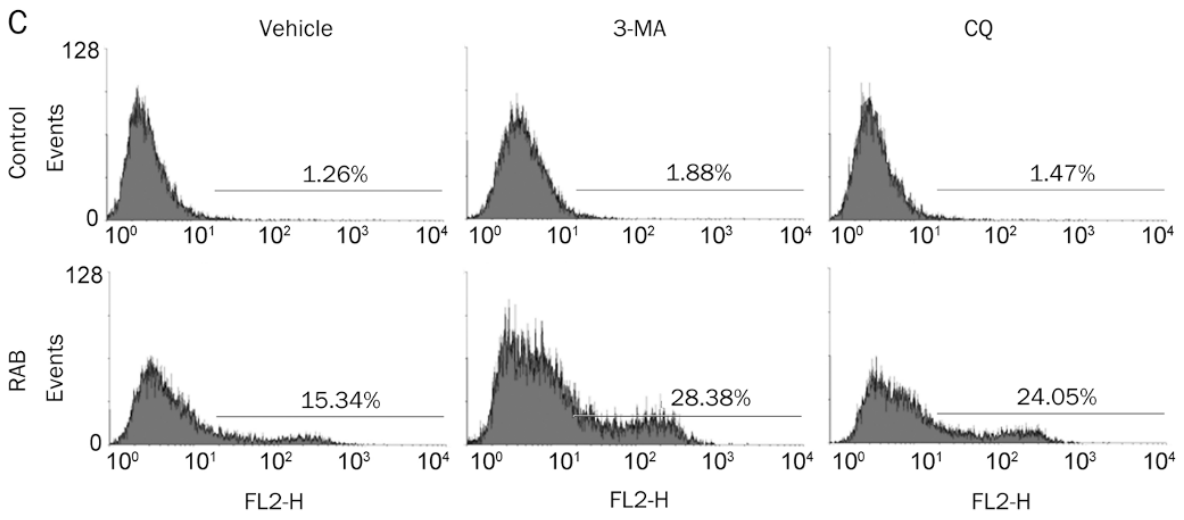

E

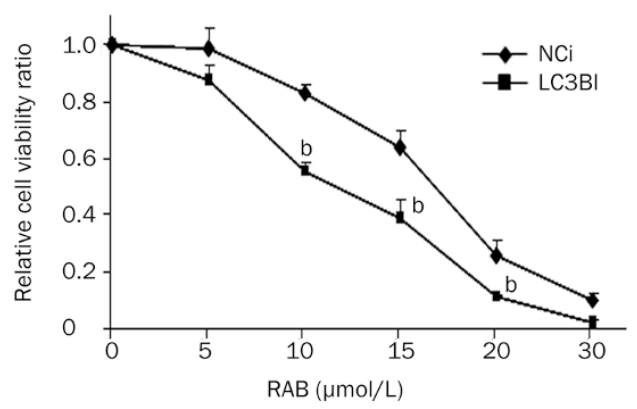

F

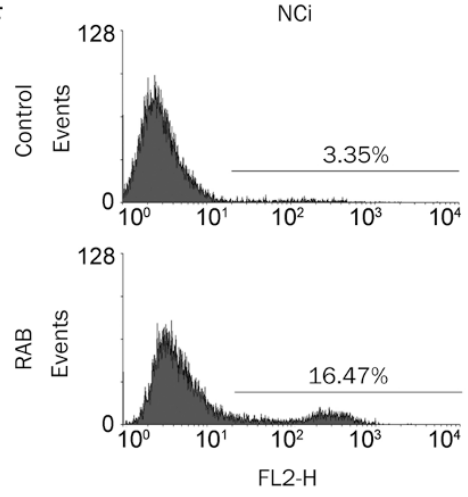

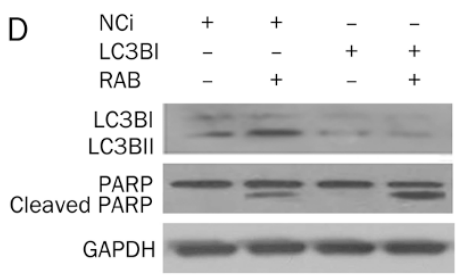

Figure 4. The blockade of autophagy enhanced RAB-mediated cell death in PCa cells. Cells were incubated with RAB in the presence or absence of 3-MA or CQ for $24 \mathrm{~h}$. PC3 cells were incubated with 3-MA $(5 \mathrm{mmol} / \mathrm{L})$ or CQ $(10 \mu \mathrm{mol} / \mathrm{L})$ for $2 \mathrm{~h}$ prior to RAB (15 $\mu \mathrm{mol} / \mathrm{L})$ treatment for $24 \mathrm{~h}$. LC3B and PARP expression were determined using Western blotting (A), and cell viability and death were measured by MTT assay (B) and PI exclusion (C), respectively. (D-F) Impact of LC3B knockdown on the cell viability of RAB-treated PC3 cells. RAB treatment was performed 24 or $48 \mathrm{~h}$ following siRNA transfection. The silencing efficiency and expression of cleaved PARP were examined using Western blotting (D). Cell viability and apoptosis rates were detected by MTT assay (E) and PI exclusion (F), respectively. GAPDH served as a loading control. ${ }^{b} P<0.05$ versus the NCi group.

and depolarized mitochondria play an essential role in the regulation of mitophagy ${ }^{[29]}$. Oxidized and damaged proteins are sequestered into mitochondria with lower membrane potential and subsequently are eliminated by mitophagy. In addition, DJ-1 can be recruited to mitochondria following oxidative stress and contribute to protection against mitochondrial toxins. However, the loss of DJ-1 leads to alterations in mitochondrial phenotypes, including reduced $\mathrm{mt} \Delta \psi$, increased fragmentation and the accumulation of autophagic markers ${ }^{[14]}$. Our data demonstrated that RAB induced oxidative stress and dramatic mitochondrial swelling with a decrease in DJ-1 expression. Together, these events promoted mitophagy in
PCa cells.

To discover the mode of mitophagy by RAB, cells were pretreated with the autophagy inhibitor 3-MA or the lysosomal protease inhibitor $C Q$, revealing that the blockade of autophagy intensified the cytotoxicity of RAB. The knockdown of LC3B also confirmed the autophagic protection. Thus, this result was consistent with the observation that the autophagy induced by RAB was subsequently followed by apoptotic cell death. As shown in Figure 2B, autophagy was detected as early as $2 \mathrm{~h}$ by the conversion of LC3BI to LC3BII following cleavage and lipid addition to LC3BI; however, after $12 \mathrm{~h}$ of RAB exposure, PARP cleavage occurred, revealing that 
apoptotic cell death had just begun, suggesting that the protective autophagy caused by RAB delayed cell death. Moreover, the expression of DJ-1 began to decline at $6 \mathrm{~h}$ and was significant until $12 \mathrm{~h}$, and its anti-apoptosis effect was lost to a great extent.

Based on the data revealed in our study, the role of RABinduced mitophagic protection in cell death was suggested to associate with the extent of mitochondrial damage. It is known that the decrease in $\mathrm{mt} \Delta \psi$ accompanying the opening of the MPT pore allows small molecular weight proteins to freely flow through the mitochondrial membrane, which deprives ATP synthase of the ability to provide sufficient energy, leading to cell death ${ }^{[24,28]}$. It has been reported that the cellular response to the MPT pore displays a mode of sequential changes: the opening of the MPT pore to increase the number of mitochondria progressively leads to autophagy, apoptosis and finally necrotic cell death ${ }^{[30,31]}$. In this study, $\mathrm{RAB}$ induced the generation of ROS, which can promote the onset of the MPT. However, onset of the MPT does not occur uniformly. Initially, just a small proportion of mitochondria underwent the MPT onset following RAB-treatment, and then the depolarized mitochondria entered an acidic lysosomal compartment as expected during mitophagy. The mitophagy induced by RAB eliminated the damaged mitochondria to protect cells from RAB-mediated oxidative stress, therefore delaying RAB-induced cell death. However, at a later stage within the 12-24 h time period, with increasing numbers of damaged mitochondria in a cell, the mitophagy protective mechanism could not control the pro-apoptosis substances, such as cytochrome $c$ and apoptosis-inducing factor, which are released from mitochondria and induce cell death. Additionally, if almost all of the mitochondria in a cell were damaged, the cell would undergo rapid necrotic cell death.

Overall, these data define a mode of action for RAB's exertion of anti-tumor activity. RAB induces ROS production, decreases $\mathrm{mt} \Delta \psi$ and DJ-1 expression, and inhibits the $\mathrm{PI} 3 \mathrm{~K} / \mathrm{Akt} / \mathrm{mTOR}$ pathway. These events strongly stimulate mitophagy, which, in turn, attenuates RAB-triggered cell death. Taken together, mitophagy may not be the direct cause of cell death, but it does represent an adaptive early response to cellular oxidative stress that could enhance cell survival by retarding apoptosis. Thus, the suppression of mitophagy might enhance the efficacy of $\mathrm{RAB}$, which could be a potential strategy for improving the chemotherapeutic effects of this compound.

\section{Acknowledgements}

This work was supported by the National Natural Science Foundation of China (81273533 and 30925038), and the Natural Science Foundation of Shandong Province, China (2009ZRB01346).

\section{Author contribution}

Hui-qing YUAN and Yong-qing LIU designed the research; Yong-qing LIU performed the experiments; Hong-xiang LOU contributed new reagents and analytic tools; Yuan JI, Xian-zhe
LI, and Ke-li TIAN analyzed the data; and Hui-qing YUAN, Charles YF YOUNG, and Yong-qing LIU wrote the paper.

\section{Abbreviations}

$R A B$, retigeric acid B; ROS, reactive oxygen species; $m t \Delta \psi$, mitochondrial transmembrane potential; MPT, mitochondrial permeability transition; 3-MA, 3-methyladenine; $\mathrm{CQ}$, chloroquine; GAPDH, glyceraldehyde-3-phosphate dehydrogenase; PARP, poly(ADP-ribose) polymerase; NAC, N-acetylcysteine; PI, propidium iodide; MTT, 3-[4,5-dimethylthiazol-2-yl]-2,5diphenyltetrazolium bromide; $\mathrm{PCa}$, prostate cancer.

\section{References}

1 Mizushima N, Levine B, Cuervo AM, Klionsky DJ. Autophagy fights disease through cellular self-digestion. Nature 2008; 451: 1069-75.

2 Yang Z, Klionsky DJ. Eaten alive: a history of macroautophagy. Nat Cell Biol 2010; 12: 814-22.

3 Tanida I, Ueno T, Kominami E. Human light chain 3/MAP1LC3B is cleaved at its carboxyl-terminal Met121 to expose Gly120 for lipidation and targeting to autophagosomal membranes. J Biol Chem 2004; 279: 47704-10.

4 Kroemer G, Marino G, Levine B. Autophagy and the integrated stress response. Mol Cell 2010; 40: 280-93.

5 Clarke R, Cook KL, Hu R, Facey CO, Tavassoly I, Schwartz JL, et al. Endoplasmic reticulum stress, the unfolded protein response, autophagy, and the integrated regulation of breast cancer cell fate. Cancer Res 2012; 72: 1321-31.

6 Nagakubo D, Taira T, Kitaura H, Ikeda M, Tamai K, Iguchi-Ariga SM, et al. DJ-1, a novel oncogene which transforms mouse NIH3T3 cells in cooperation with ras. Biochem Biophys Res Commun 1997; 231: 509-13.

7 Shendelman S, Jonason A, Martinat C, Leete T, Abeliovich A. DJ-1 is a redox-dependent molecular chaperone that inhibits alpha-synuclein aggregate formation. PLoS Biol 2004; 2: e362.

8 Lev N, Ickowicz D, Barhum Y, Lev S, Melamed E, Offen D. DJ-1 protects against dopamine toxicity. J Neural Transm 2009; 116: 151-60.

9 Cookson MR. Pathways to Parkinsonism. Neuron 2003; 37: 7-10.

10 Liu H, Wang M, Li M, Wang D, Rao Q, Wang Y, et al. Expression and role of DJ-1 in leukemia. Biochem Biophys Res Commun 2008; 375: 477-83.

11 Mackeigan JP, Clements CM, Lich JD, Pope RM, Hod Y, Ting JP. Proteomic profiling drug-induced apoptosis in non-small cell lung carcinoma: identification of RS/DJ-1 and RhoGDlalpha. Cancer Res 2003; 63: 6928-34.

12 Ismail IA, Kang HS, Lee HJ, Kwon BM, Hong SH. 2'-Benzoyloxycinnamaldehyde-mediated DJ-1 upregulation protects MCF-7 cells from mitochondrial damage. Biol Pharm Bull 2012; 35: 895-902.

13 Junn E, Jang WH, Zhao X, Jeong BS, Mouradian MM. Mitochondrial localization of DJ-1 leads to enhanced neuroprotection. J Neurosci Res 2009; 87: 123-9.

14 McCoy MK, Cookson MR. DJ-1 regulation of mitochondrial function and autophagy through oxidative stress. Autophagy 2011; 7: 531-2.

15 Taira T, Saito Y, Niki T, Iguchi-Ariga SM, Takahashi K, Ariga H. DJ-1 has a role in antioxidative stress to prevent cell death. EMBO Rep 2004; 5 : 213-8.

16 Opipari AW Jr, Tan L, Boitano AE, Sorenson DR, Aurora A, Liu JR. Resveratrol-induced autophagocytosis in ovarian cancer cells. Cancer Res 2004; 64: 696-703.

17 O'Donovan TR, O'Sullivan GC, McKenna SL. Induction of autophagy 
by drug-resistant esophageal cancer cells promotes their survival and recovery following treatment with chemotherapeutics. Autophagy 2011; 7: 509-24.

18 Viola G, Bortolozzi R, Hamel E, Moro S, Brun P, Castagliuolo I, et al. MG-2477, a new tubulin inhibitor, induces autophagy through inhibition of the Akt/mTOR pathway and delayed apoptosis in A549 cells. Biochem Pharmacol 2012; 83: 16-26.

19 Xi G, Hu X, Wu B, Jiang H, Young CY, Pang Y, et al. Autophagy inhibition promotes paclitaxel-induced apoptosis in cancer cells. Cancer Lett 2011; 307: 141-8.

20 Abedin MJ, Wang D, McDonnell MA, Lehmann U, Kelekar A. Autophagy delays apoptotic death in breast cancer cells following DNA damage. Cell Death Differ 2007; 14: 500-10.

21 Liu YQ, Hu XY, Lu T, Cheng YN, Young CY, Yuan HQ, et al. Retigeric acid $B$ exhibits antitumor activity through suppression of nuclear factorkappaB signaling in prostate cancer cells in vitro and in vivo. PLoS One 2012; 7: e38000.

22 Liu H, Liu YQ, Liu YQ, Xu AH, Young CY, Yuan HQ, et al. A novel anticancer agent, retigeric acid $\mathrm{B}$, displays proliferation inhibition, $\mathrm{S}$ phase arrest and apoptosis activation in human prostate cancer cells. Chem Biol Interact 2010; 188: 598-606.

23 Yuan H, Young CY, Tian Y, Liu Z, Zhang M, Lou H. Suppression of the androgen receptor function by quercetin through protein-protein interactions of Sp1, c-Jun, and the androgen receptor in human prostate cancer cells. Mol Cell Biochem 2010; 339: 253-62.
24 Zoratti M, Szabo I. The mitochondrial permeability transition. Biochim Biophys Acta 1995; 1241: 139-76.

25 Takeuchi H, Kondo Y, Fujiwara K, Kanzawa T, Aoki H, Mills GB, et al. Synergistic augmentation of rapamycin-induced autophagy in malignant glioma cells by phosphatidylinositol 3-kinase/protein kinase B inhibitors. Cancer Res 2005; 65: 3336-46.

26 VanderWeele DJ, Zhou R, Rudin CM. Akt up-regulation increases resistance to microtubule-directed chemotherapeutic agents through mammalian target of rapamycin. Mol Cancer Ther 2004; 3: 1605-13.

27 Singh K, Matsuyama S, Drazba JA, Almasan A. Autophagy-dependent senescence in response to DNA damage and chronic apoptotic stress. Autophagy 2012; 8: 236-51.

28 Ferrin G, Linares $\mathrm{Cl}$, Muntane J. Mitochondrial drug targets in cell death and cancer. Curr Pharm Des 2011; 17: 2002-16.

29 Li ZY, Yang Y, Ming M, Liu B. Mitochondrial ROS generation for regulation of autophagic pathways in cancer. Biochem Biophys Res Commun 2011; 414: 5-8.

30 Lemasters JJ, Qian T, Elmore SP, Trost LC, Nishimura Y, Herman B, et al. Confocal microscopy of the mitochondrial permeability transition in necrotic cell killing, apoptosis and autophagy. Biofactors 1998; 8: 283-5.

31 Lemasters JJ, Nieminen AL, Qian T, Trost LC, Elmore SP, Nishimura $\mathrm{Y}$, et al. The mitochondrial permeability transition in cell death: a common mechanism in necrosis, apoptosis and autophagy. Biochim Biophys Acta 1998; 1366: 177-96. 